\title{
APPLICATION OF GEOGEBRA FOR TEACHING MATHEMATICS
}

\author{
Dariusz Majerek ${ }^{1}$ \\ 1 Department of Applied Mathematics, Lublin University of Technology, Nadbystrzycka 38, 20-618 Lublin, \\ Poland, e-mail: d.majerek@pollub.pl
}

Received: 2014.09.25

Accepted: 2014.10.25

Published: 2014.12.01

\begin{abstract}
This paper shows how GeoGebra can be helpful in teaching mathematics. GeoGebra is an interactive geometry, algebra, statistics and calculus application, intended for learning and teaching mathematics and science from primary school to university level. It can be used for active and problem oriented teaching and fosters mathematical experiments and discoveries both in classroom and at home. In this work we show the sketch of using the above-mentioned software to build, solve and illustrate mathematical problems.
\end{abstract}

Keywords: dynamic geometry, algebra, calculus.

\section{INTRODUCTION}

Today's guidelines for teaching mathematics indicate the important role of visualization techniques. As a response for these needs many of software application were created to build geometric constructions and solving analytical and algebraic problems. One of the best application designed to construct and illustrate some mathematical issues is GeoGebra.

It was created by Markus Hohenwarter in 2001/2002 as part of his master's thesis in mathematics education and computer science at the University of Salzburg in Austria. Supported by the Austrian Academy of Science he was able to develop the software as a part of his $\mathrm{PhD}$ project in mathematics education. Meanwhile GeoGebra received many international awards, and was translated by mathematics instructors and teachers all over the world to more than 25 languages. Since 2006 GeoGebra is supported by the Austrian Ministry of Education to maintain the free availability of the software for mathematics education at schools and universities. In July 2006, GeoGebra found its way to the US, where its development continues at Florida Atlantic University in the NSF project Standard Mapped Graduate Education and Mentoring. [1]
GeoGebra depends on software licensed under the GNU General Public License (GPL), the LGPL, the Apache license and others. The software is licensed under the «GeoGebra NonCommercial License Agreement», which asserts that while the «source code is licensed [...] under the terms of the GNU General Public License», the translation files, installers, and web services are licensed under non-GPL-compatible terms. Commercial use is prohibited without the purchase of a separate license which prevents the resulting combined work from being considered free software. [2]

GeoGebra is available on multiple platforms with its desktop applications for Windows, Mac OS and Linux, with its tablet apps for Android, iPad and Windows, and with its web application based on HTML5 technology.

\section{THE MAIN IMPEDIMENTS IN TEACHING MATHEMATICS}

Today young people are accustomed to pictorial culture through a wide access to social media such as facebook, twitter or youtube. A multitude of different forms of media are involved, including, for example text, audio, graphics, animation, video, and virtual reality. Additionally, 
the development and rapid growth of the Internet in combination with its increasing accessibility for the public has opened up a whole new digital world. This makes that in learning process students are increasingly inclined to accept the content given to them in this way. Especially in mathematics where a number of issues require a lot of imagination, students are discouraged to learn the subject when it is not provided in a modern and accessible way.

The main obstacles in teaching mathematicsematics are:

- Concepts without an adequate illustration,

- Mathematical graphs are static in classical way of teaching mathematics i.e. drawing it on a piece of paper,

- Static objects do not allow for generalization of the concept.

The National Council of Teachers of Mathematics (NCTM), which is the world's largest association of mathematics teachers, declared technology as one of their six principles for school mathematics.

Technology is essential in teaching and learning mathematics; it influences_the mathematics that is taught and enhances students' learning. [3]

\section{MAIN FEATURES OF GEOGEBRA}

he main idea of using GeoGebra into everyday teaching and learning is to provide opportunities for students of different mathematical skills and levels for better understanding concepts and fostering them to doing mathematics in new attractive way.

Here are the main features of Geogebra:

- free for noncommercial use,

- multiplatform,

- clear and easy understanding graphical user interface,

- rich database of ready-made examples,

- technical documentation in many languages,

- marking objects follow the mathematical syntax,

- ability to save a project in multiple formats,

- works with LaTeX,

- all objects in GeoGebra are dynamic,

- possibility to publish the work on the website through javascript,

- program is translated into many foreignlanguages.
All this makes the GeoGebra a great tool for teaching and learning mathematics. Since all objects in GeoGebra are dynamic, students can see how it's changing when he change the parameters of the problem. In geometrical constructions all the objects such as points, sections, circles and lines can be moved in any way. This makes the constructions more clearer. In addition all the constructions can be made by point and click technique or introducing them through command line.

\section{INTERFACE}

GeoGebra has a very clear and intuitive interface divided into parts corresponding to the algebra and geometry (Figure 1). Depending on your needs it can be freely modified to suit the considered issue.

We have several views:

- algebraic view,

- geometric view,

- spreadsheet view,

- CAS (Computer Algebra System) view,

- protocol design view,

- command line.

All these views are linked with each other, that is, if we introduce an object in one of the views, it will appear in others in an appropriate form. So for example, if we put a function in a command line, its graph appears in geometric view. All the changes of the parameters of the function are immediately shown on graph.

Beside the basic capabilities of GeoGebra like drawing figures, lines and function graphs we can also calculate or measure angles, points of interceptions, lengths, fields, circumferences, maximum and minimum of a function, derivatives and integrals. Obviously, GeoGebra can be used as an advanced calculator but not only. It can operate on vectors, matrices and even solve a system of linear equations.

\section{EXAMPLES}

We show the capabilities of GeoGebra in some examples.

\section{Example 1}

Suppose we want to construct a circle described on the triangle (Figure 2). We know that 


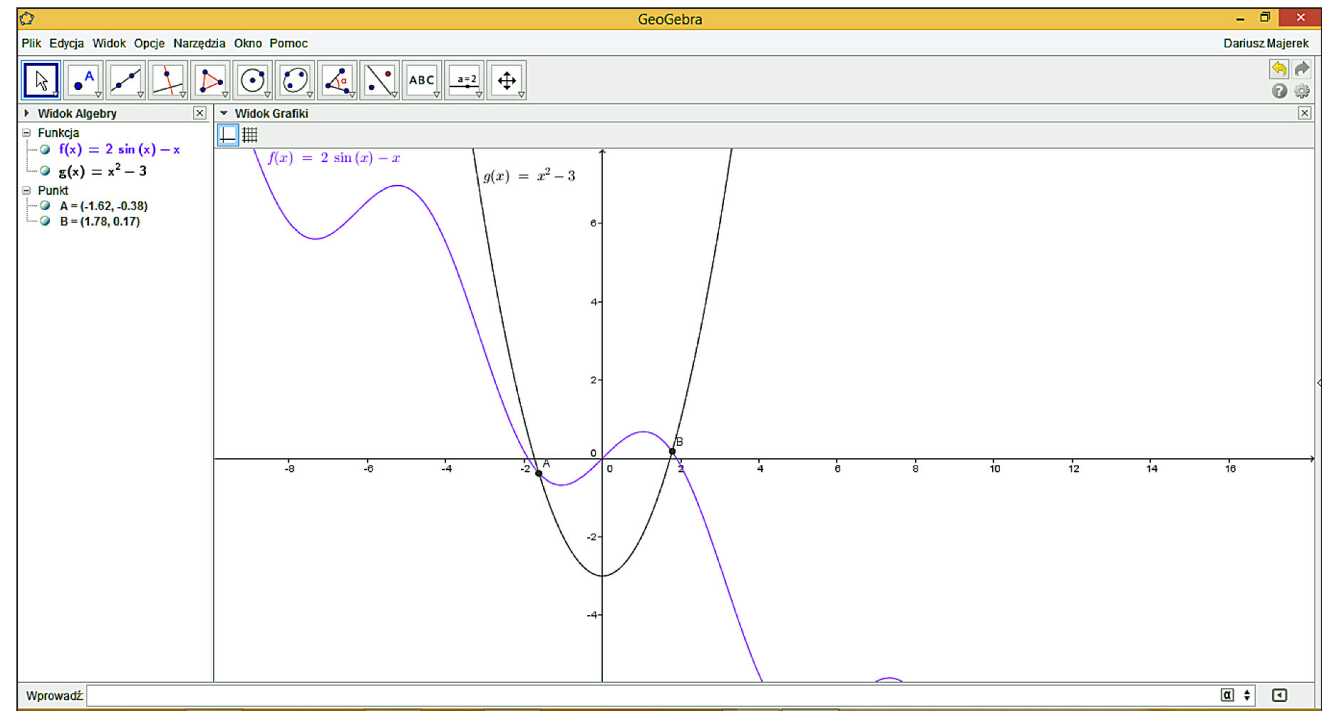

Fig. 1. GeoGebra interface

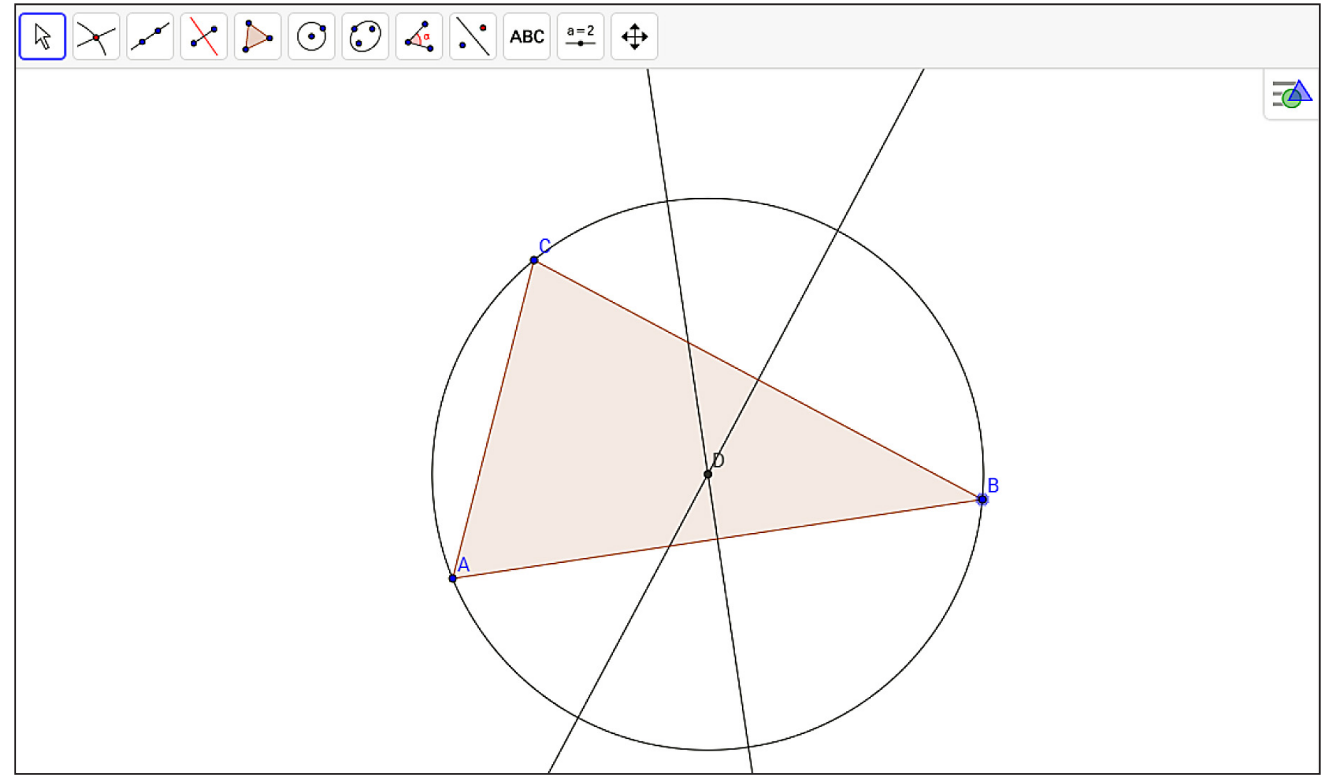

Fig. 2. Circle described on a triangle

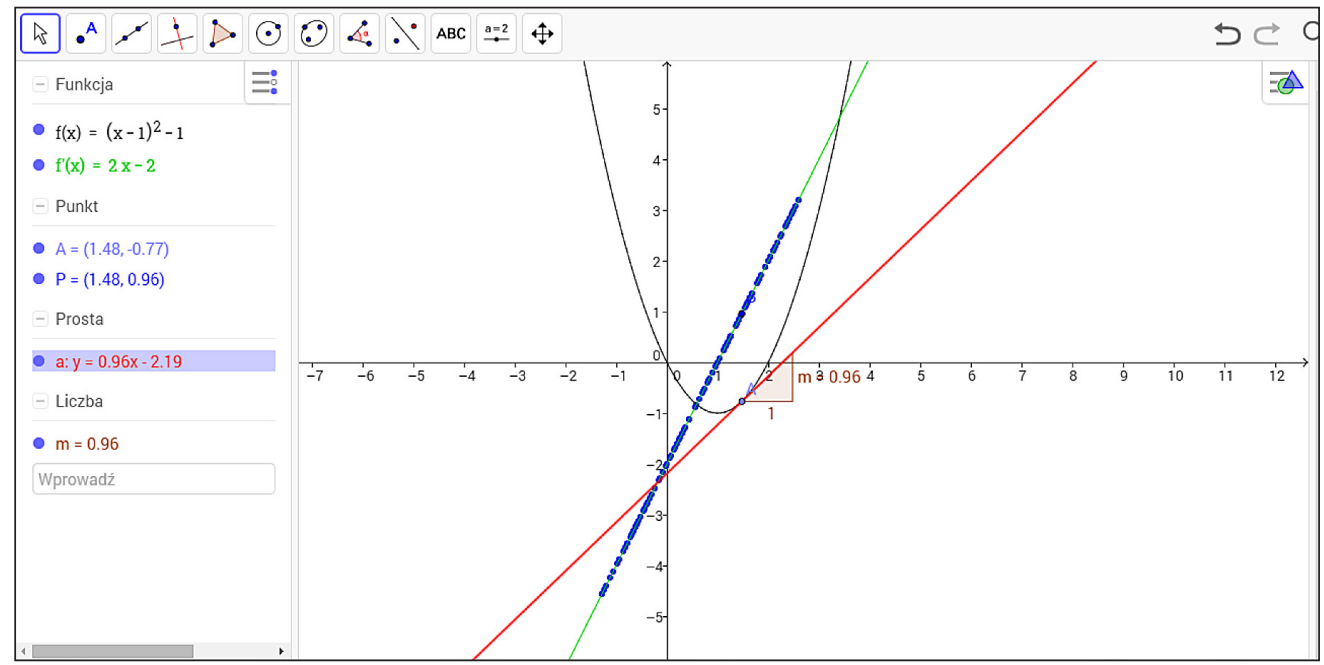

Fig. 3. Tangent line and derivative of the function 
the center of the circle must be an interception of bisectors. Radius of the circle is the sector from intersection of two bisectors to one of the vertex. Construction of a circle is performed in the following steps:

- draw any triangle $A B C$,

- construct two bisectors of any two sides,

- find the interception of bisectors and mark it by $D$,

- draw a circle with center in $D$ and radius $\overline{D A}$.

\section{Example 2}

In this example we want to show the relationship between the slope of a tangent line of a function and derivative of this function. In the first step we have to draw some function, for example:

$$
f(x)=(x-1)^{2}-1 .
$$

The next step is to put a point on the graph of $f$ called $A$. Then draw a tangent line in point $A$ (Figure 3). This line has slope and we call it $m$. If we put a point $P=(x(A), m)$ on a graph and set the option of this point to do trace if it moves, then together with point $A$ point $P$ will also be moved and trace a line which is identical with derivative of $f$ given by formula:

$$
f^{\prime}(x)=2 x-2
$$

This example helps students understand the meaning of the derivative of a function. Obviously we can change the formula of $f$ and then all points and lines changes automatically, so student can see this phenomenon for different functions.

\section{CONCLUSION}

We show the benefits of introduction dynamic mathematics software into teaching and learning process. We conclude that all students, from any level of mathematical knowledge can be encouraged to study mathematics by using this application. Current trends in the teaching of science call for the use of visualization techniques, and GeoGebra fits perfectly this trend.

\section{REFERENCES}

1. Hohenwarter M., Preiner J.: Dynamic mathematics with GeoGebra. The Journal of Online Mathematics and Its Applications, No. 7, 2007.

2. International GeoGebra Institute, ,GeoGebra" [Available: https://www.geogebra.org/license, 15.10.2014].

3. NCTM, „Principles and Standards for School Mathematics", NCTM, 2000. 